\title{
Article \\ Noncured Graphene Thermal Interface Materials for High-Power Electronics: Minimizing the Thermal Contact Resistance
}

\author{
Sriharsha Sudhindra *(D), Fariborz Kargar*(D) and Alexander A. Balandin * $+\mathbb{D}$ \\ Phonon Optimized Engineered Materials Center, Department of Electrical and Computer Engineering, \\ University of California, Riverside, CA 92521, USA \\ * Correspondence: ssudh002@ucr.edu (S.S.); fkargar@ece.ucr.edu (F.K.); balandin@ece.ucr.edu (A.A.B.) \\ + Web-Site: http://balandingroup.ucr.edu/.
}

check for

updates

Citation: Sudhindra, S.; Kargar, F.; Balandin, A.A. Noncured Graphene Thermal Interface Materials for High-Power Electronics: Minimizing the Thermal Contact Resistance. Nanomaterials 2021, 11, 1699. https://doi.org/10.3390/nano 11071699

Academic Editors: Elena Sheka, Tai-Feng Hung and Yanfeng Gao

Received: 3 June 2021

Accepted: 25 June 2021

Published: 28 June 2021

Publisher's Note: MDPI stays neutral with regard to jurisdictional claims in published maps and institutional affiliations.

Copyright: (c) 2021 by the authors. Licensee MDPI, Basel, Switzerland. This article is an open access article distributed under the terms and conditions of the Creative Commons Attribution (CC BY) license (https:// creativecommons.org/licenses/by/ $4.0 /)$.

\begin{abstract}
We report on experimental investigation of thermal contact resistance, $R_{C}$, of the noncuring graphene thermal interface materials with the surfaces characterized by different degree of roughness, $S_{q}$. It is found that the thermal contact resistance depends on the graphene loading, $\xi$, non-monotonically, achieving its minimum at the loading fraction of $\xi \sim 15 \mathrm{wt} \%$. Decreasing the surface roughness by $S_{q} \sim 1 \mu \mathrm{m}$ results in approximately the factor of $\times 2$ decrease in the thermal contact resistance for this graphene loading. The obtained dependences of the thermal conductivity, $K_{T I M}$, thermal contact resistance, $R_{C}$, and the total thermal resistance of the thermal interface material layer on $\xi$ and $S_{q}$ can be utilized for optimization of the loading fraction of graphene for specific materials and roughness of the connecting surfaces. Our results are important for the thermal management of high-power-density electronics implemented with diamond and other wide-band-gap semiconductors.
\end{abstract}

Keywords: surface roughness; thermal contact resistance; thermal conductivity; graphene; silicone oil; thermal interface materials

\section{Introduction}

A continuing trend of the miniaturization of electronic devices for information processing [1-4], and the increasing power density in high-power electronics [5-10] dictate the need for more efficient thermal management [11]. The reliability of devices and systems depend on their operating temperature [12]. Increasing device temperature results in an exponential increase in the rate of device failure $[5,13]$. Thermal interface materials (TIMs) are applied between the device and the heat spreader or heat sink to facilitate the heat transfer from the heat source to the environment (see Figure 1). Typically, less than $2 \%$ of the overall area interacts with each other when two surfaces, metallic or semiconductor, are placed in contact $[14,15]$. The remaining area is occupied by air, which has a low thermal conductivity of $0.026 \mathrm{Wm}^{-1} \mathrm{~K}^{-1}$ at room temperature (RT) [16]. Filling the air gaps with TIMs that have substantially larger thermal conductivity comparing to that of the air is the main strategy for conventional thermal management approaches. Development of more efficient TIMs that can provide smaller thermal resistance for heat escape has become an important goal for electronic industry, and particularly for its segment, which deals with the high-power devices and systems [5,14,17].

The efficiency of the TIM connecting two surfaces is define by the total thermal resistance [18-20]:

$$
R_{t o t}=B L T / K_{T I M}+R_{C 1}+R_{C 2} .
$$

Here, $K_{T I M}$ is the thermal conductivity of the TIM, $R_{C 1}$ and $R_{C 2}$ are the thermal resistances of the TIM layer with the two contact surfaces, and BLT is the bond line thickness, which is the thickness of the TIM layer. The BLT/K $K_{T I M}$ represents the ther- 
mal resistance of the TIM layer. If TIM is used with the two identical surfaces, then $R_{C 1}=R_{C 2}=R_{C}$ and Equation (1) is simplified to:

$$
R_{t o t}=B L T / K_{T I M}+2 R_{C} \text {. }
$$

Minimizing $B L T$ and $R_{C}$ reduces the overall thermal resistance, $R_{t o t}$. These parameters depend on the thermophysical properties of the interlayer TIM and the roughness of the surfaces in contact. Roughness is determined by the nanoscale and microscale variations in the height profile of the physical surface. Typically, in modern electronics, BLT is assumed to vary from 25 to $100 \mu \mathrm{m}[5,21]$. For the thermal management of high-power-density electronics one may need larger BLT owing to the possible increase of roughness of the surfaces. For example, polycrystalline diamond, which can be used either as a substrate or active device layers is often characterized by large roughness due to the grains [22,23]. While many reports on new TIMs focus on the increase of the thermal conductivity, $K_{T I M}$, of the TIM composite, one should note from the above equation for $R_{t o t}$, that the improvement of thermal management requires that TIM interfaces well with given surfaces, resulting in smaller $R_{C}$, and that BLT is optimized for a given surface roughness.

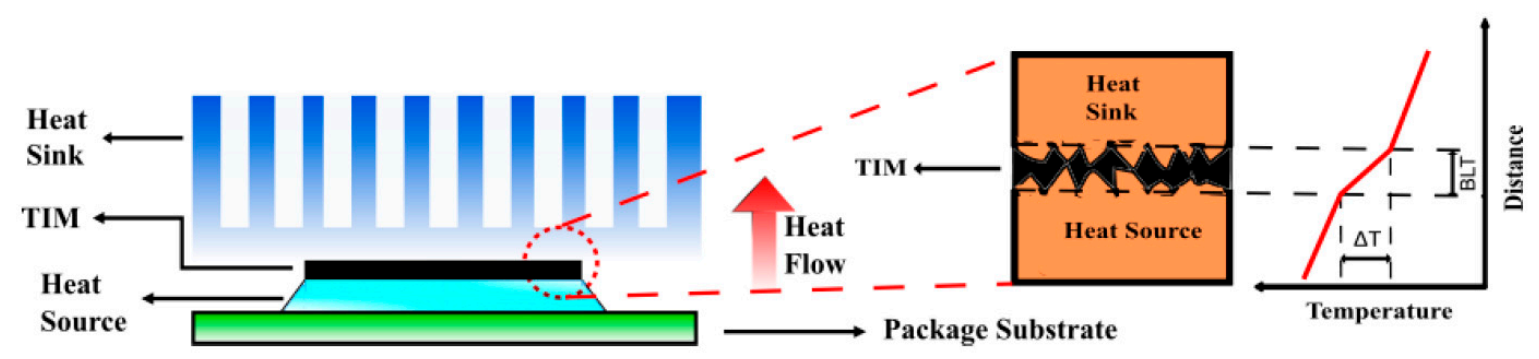

Figure 1. Schematic representation of a packaged device illustrating the role of TIMs. TIM is applied between the adjoining heat source and the heat sink surfaces in order to fill the air gaps and facilitate the heat transfer.

The main strategy for improvement of TIMs is incorporating thermally conductive fillers into the base polymer matrix, which can increase the overall thermal conductivity, $K_{T I M}$, of the TIM composites without substantially increasing $R_{C}$. In recent years, graphene has revealed its potential as a filler for both curing TIMs, e.g., with epoxy base, and noncuring TIMs, e.g., with silicone or other mineral oil bases. Graphene has extremely high intrinsic thermal conductivity, exceeding that of bulk graphite, which is $\sim 2000 \mathrm{Wm}^{-1} \mathrm{~K}^{-1}$ near RT [24-26]. It was also established that few-layer graphene (FLG) maintains high thermal conductivity, similar to bulk graphite owing to its smooth surface and, as a result, insignificant reduction in thermal conductivity due to the phonon-boundary scattering [27-30]. A mixture of single-layer graphene and FLG demonstrated the largest enhancement in the thermal conductivity of the TIM composites [19,20,31-49]. In the context of thermal research and TIMs, we will refer to the processed mixture of graphene and FLG flakes with lateral dimensions in several $\mu \mathrm{m}$ range as graphene fillers. The thickness of the FLG fillers should be in the nanometer-scale range to preserve their flexibility. Such fillers can be produced inexpensively on a large industrial scale. The latter makes graphene TIMs much more practical than any composites with carbon nanotubes or other expensive materials.

Most of prior works on graphene TIMs report the thermal conductivity values of the composites and, in some cases, temperature rise experiments with specific device structures [34,50-52]. The questions of the thermal contact resistance of graphene TIMs with the surfaces of interest and the effects of roughness on the TIM performance have not been properly addressed. These are important issues for minimizing $R_{t o t}$ for different electronic applications, particularly for the high-power density electronics where the surfaces can be characterized by larger roughness and hence, higher $R_{C}$. Here, we investigate the thermal contact resistance of the noncuring graphene TIMs with the surfaces characterized 
by different degree of roughness. The dependence of the total thermal resistance of the noncuring graphene TIMs on BLT is also obtained. In Section 2, we present experimental procedures. The discussion and conclusions are given in Sections 3 and 4, respectively.

\section{Synthesized Samples and Experimental Procedures}

For this study, we used noncuring silicone-oil based TIMs with graphene and FLG fillers prepared from the commercial graphene powder (xGNP H-25, XG Sciences, Lansing, MI, USA, NAM). The noncuring graphene TIMs were applied to copper square plates (Midwest Steel Supply, Rogers, MN, USA, NAM) of thickness $1.09 \mathrm{~mm}$ and dimensions of 1 in $\times 1$ in. The copper plates were polished (Allied High-Tech Products, Inc., Compton, CA, USA, NAM) and then treated with the sand paper to a different degree of roughness. A 3D optical profilometer (Profilm 3D, Filmetrics Inc., San Diego, CA, USA, NAM) was used to determine quantitatively the surface roughness values of the copper plates. The optical profiler utilized in this work operates on the basis of the non-contact optical technique of the white-light interferometry (WLI) plates [53]. The details of the preparation of noncuring graphene TIMs and surface treatment of the copper plates are described in the Methods Section. Figure 2 shows the results of the profilometer measurements for a set of copper plates. Figure $2 \mathrm{a}$ is the roughness of reference copper plate as received from the vendor. The sample was not polished by the polisher. To increase the surface roughness of the copper plates shown in Figure $2 b-d$, the plates were polished at 100 RPM for $\sim 1, \sim 2.5$ and $\sim 3.5 \mathrm{~min}$, respectively. The areal root mean square (RMS) roughness, $S_{q}$, determined for these plates was $0.05,1.2,2.5$ and $3.1 \mu \mathrm{m}$, respectively. The preparation of the surfaces and the profilometer measurements allowed us to investigate the effect of roughness on thermal contact resistance with graphene TIMs.
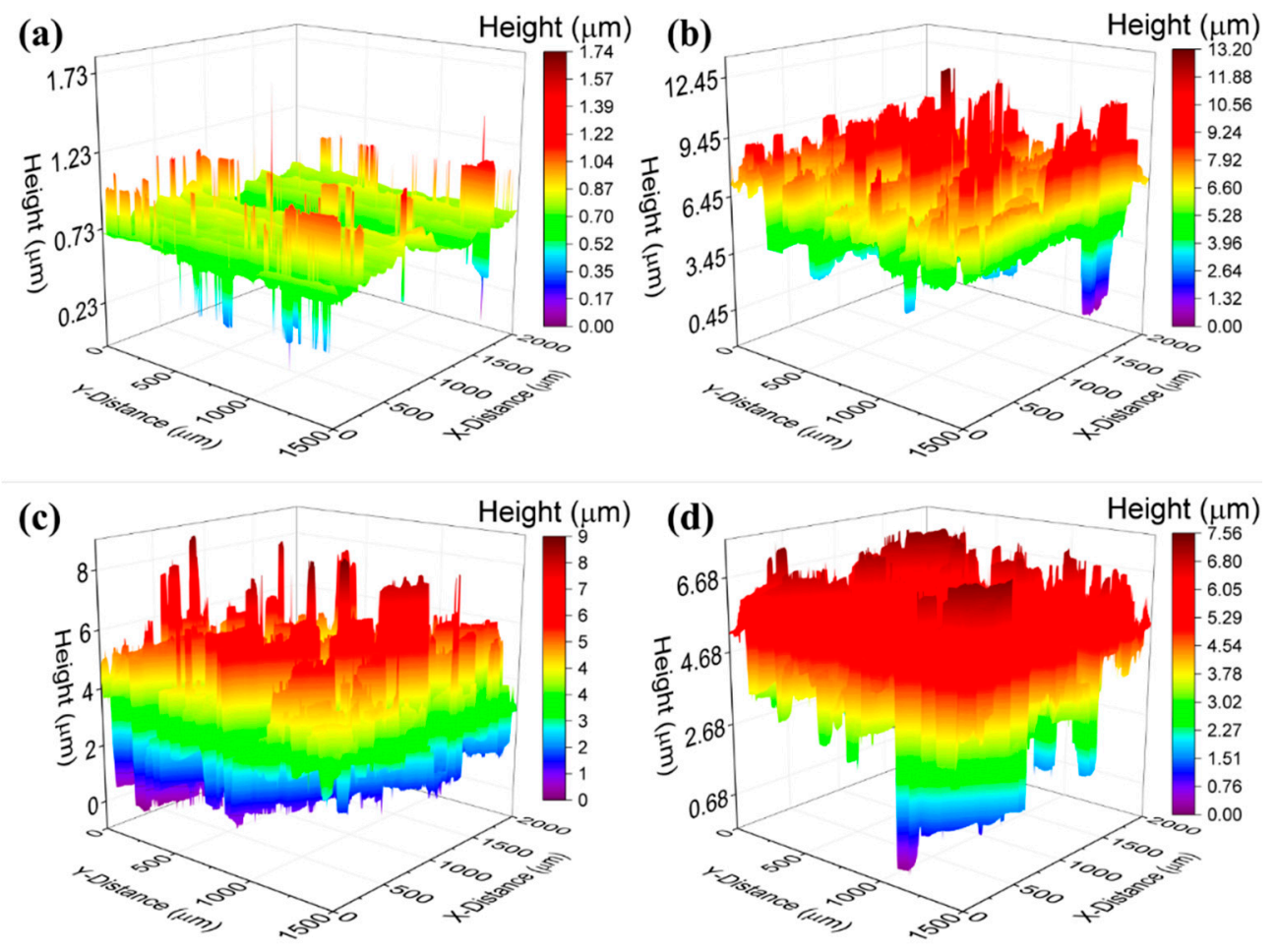

Figure 2. Roughness characteristics of the copper plates determined by an optical profilometer. The plates have the following root mean square (RMS) roughness: (a) $S_{q}=0.05 \mu \mathrm{m}$, (b) $S_{q}=1.2 \mu \mathrm{m}$, (c) $S_{q}=2.5 \mu \mathrm{m}$, and (d) $S_{q}=3.1 \mu \mathrm{m}$.

Bulk thermal conductivity, total thermal resistance, $R_{t o t}$, and thermal contact resistance of the TIM with the surface of the plates, $R_{C}$, were measured following the ASTM D5470-06 standard with an industrial TIM tester (LongWin Science and Technology Corp, Taiwan). 
The schematic of the measurement setup is shown in Figure S1 in Supplementary Information. The TIM tester utilizes the steady-state method [54]. The measurement setup is comprised of two very flat steel plates with roughness in the range of a few $\mathrm{nm}$ as the heat source and sink. The TIM is applied between these plates. The heat flow and the temperature of the source and sink are carefully controlled. The thermal conductivity of TIM is extracted using the one-dimensional Fourier heat transport equation for given BLT of TIM. The details of the thermal testing are provided in the Methods Section. The initial measurements were performed on TIMs with different loading of graphene content without the copper plates. A layer of the synthesized TIM was applied between the two plates of the TIM tester. The $B L T$ was controlled using the plastic shims. Note that the shims occupy a negligible portion of the area and volume of the TIM material that their contribution to overall heat transfer is negligible. All measurements have been performed under $0.55 \mathrm{MPa}(\sim 80 \mathrm{psi})$ of applied pressure, $P$.

\section{Results and Discussion}

We first measured the thermal properties of the prepared non-cured graphene TIMs without the copper plates. Figure $3 a, b$ shows the total thermal resistance of graphene TIM, $R_{t o t}$, as a function of BLT for different graphene loading, $\xi$. Figure 3a includes the thermal resistance of the silicone oil base as a reference. Figure $3 b$ shows the data for the graphene loading of $10 \mathrm{wt} \%$ and more so that the trends can be seen more clearly. The total thermal resistance increases linearly with $B L T$ as expected $[55,56]$. The data were used to plot a linear regression fitting for each loading fraction. For each fitting, the inverse of the line slope determines the bulk thermal conductivity of the TIM itself. The $y$-intercept of the fitted line is equal to the total thermal contact resistance, $2 R_{C}$, of each TIM with the upper and lower contact surfaces. A table showing the obtained values is provided in the Supporting Information (Table S1).

Figure 4 shows the thermal conductivity of the noncuring graphene TIMs as a function of graphene loading, $\xi$. The thermal conductivity of the silicon oil base is $0.18 \mathrm{Wm}^{-1} \mathrm{~K}^{-1}$. The thermal conductivity starts to increase rapidly with the addition of graphene. The increase is super-linear suggesting that the fillers form a percolated network facilitating the heat conduction. Note that in this Figure the $y$-axis is in logarithmic scale. At the loading of $\xi=10 \mathrm{wt} \%$, the increase in thermal conductivity slows down. This trend is consistent with prior studies for noncuring graphene composites [41], and different from that observed in curing epoxy composites with graphene [19,20,36-40,42]. In the cured solid TIMs, the thermal conductivity reveals linear to super-linear dependence on the filler loading [39]. The non-curing TIMs, on the other hand, exhibit a saturation effect for the thermal conductivity starting at some critical filler loading. This is similar to the effect reported previously for nano-fluids and soft TIMs [57-60]. The saturation effect can be explained by the tradeoff between the enhancement trend in the thermal conductivity as more fillers are added to the matrix and the decrease in the thermal conductance as the thermal interface resistance between the filler-filler and filler-matrix interfaces increases due to the incorporation of more fillers into the matrix [41]. In our noncuring TIMs, we achieved the value of the thermal conductivity of $\sim 4.2 \mathrm{Wm}^{-1} \mathrm{~K}^{-1}$ at the graphene loading of $40 \mathrm{wt} \%$. We intentionally did not increase the loading further due to the onset of the agglomeration. For the purpose of this study, it was important to have the consistent dispersion of the fillers. Overall, the thermal conductivity of graphene TIMs increased by the factor of $\sim 19 \times$ for $30 \mathrm{wt} \%$ and $24 \times$ for $40 \mathrm{wt} \%$ loadings compared to the thermal conductivity of the silicone oil base.

In Figure 5, we present the measured thermal contact resistance of the TIM, $R_{C}$, as a function of graphene loading, $\xi$. $R_{C}$ is obtained by dividing the $y$-intercept of the fitted lines in Figure 3 by two (see Equation (2)). The measured $R_{C}(\xi)$ dependence revealed a rather unexpected non-monotonic trend. Contrary to the expectation of increasing $R_{C}$ with higher filler loading, we observe a rapid decrease in $R_{C}$ values up to the loading $\xi=15 \mathrm{wt} \%$, followed by a slow increase at the higher loading fraction. Theoretically, $R_{C}$ depends on 
the bulk thermal conductivity and shear modulus of the TIM and the roughness of the adjoining surfaces and the applied pressure. There is a trade-off between the thermal conductivity and shear modulus effect on $R_{C}$. The higher is the thermal conductivity, the lower is $R_{C}$, whereas for the shear modulus the dependence is vice versa [61]. Typically, one would want to increase the loading to improve $K_{T I M}$ as long as the viscosity and the shear modulus requirements allow for it. Based on the measured $R_{C}(\xi)$ dependence, one may prefer to limit the loading to smaller fraction in order to minimize $R_{t o t}$. One should also note that increasing $\xi$ limits the minimum attainable BLT.
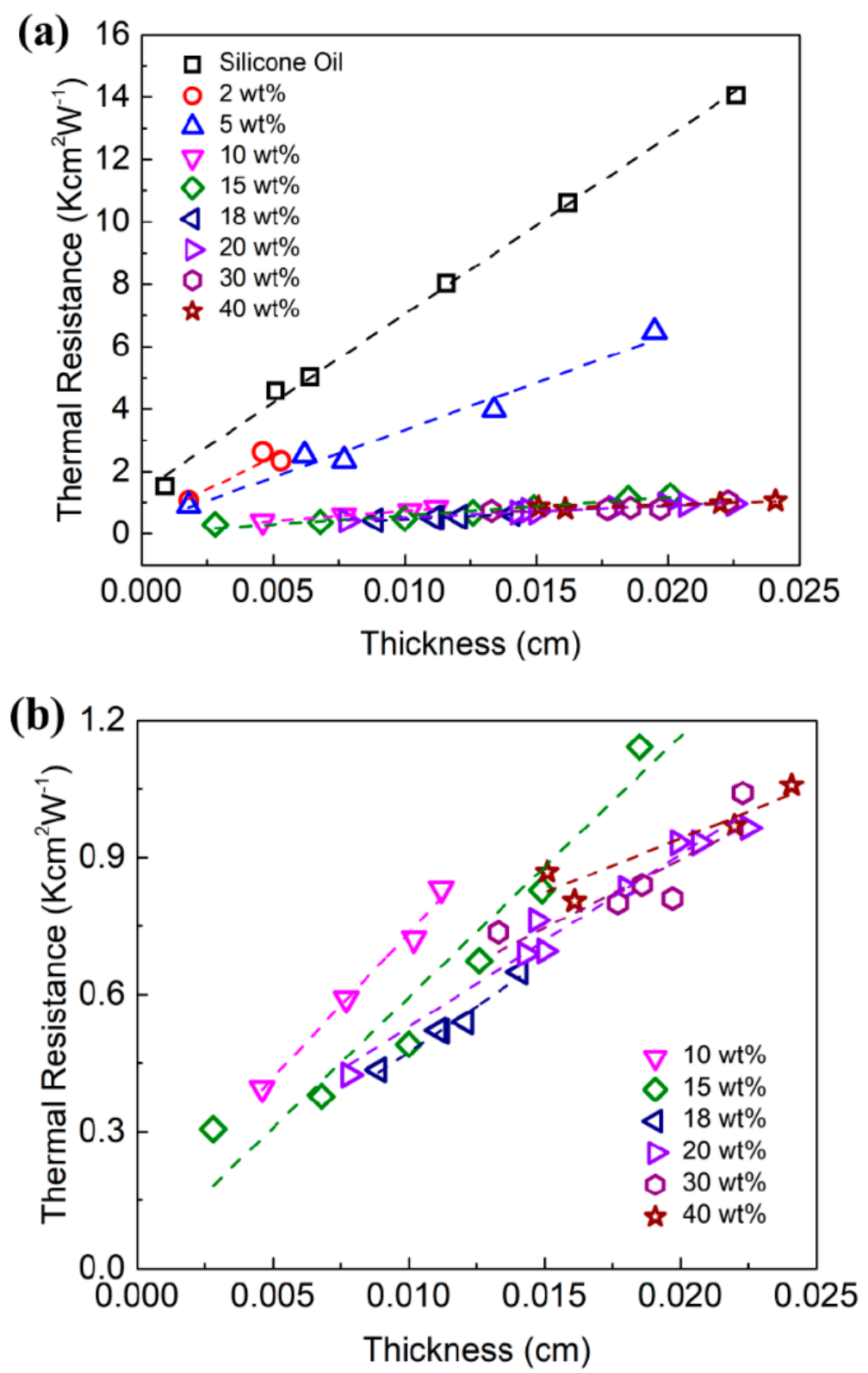

Figure 3. Total thermal resistance, $R_{t o t}$, of graphene TIMs as a function of the bond line thickness, $B L T$. The dashed lines show the linear regression to the experimental data used to extract the thermal conductivity, $K_{T I M}$, and thermal contact resistance, $R_{C}$. (a) Thermal resistance vs. BLT for all tested loading fractions of graphene and pure silicone oil base. (b) Thermal resistance vs. BLT for loading fractions of $\xi=10 \mathrm{wt} \%$ and above are shown for clarity. 


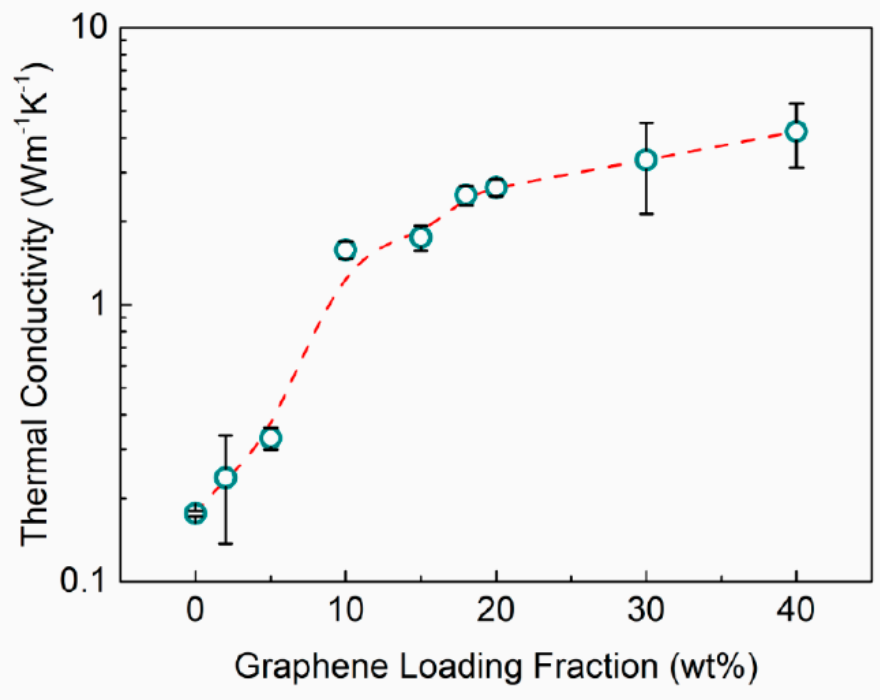

Figure 4. Thermal conductivity, $K_{T I M}$, of TIMs as a function of graphene loading fraction, $\xi$. Adding graphene fillers to the noncuring oil base increases the "bulk" thermal conductivity of graphene TIMs. The bars show the standard error of the linear regression slope.

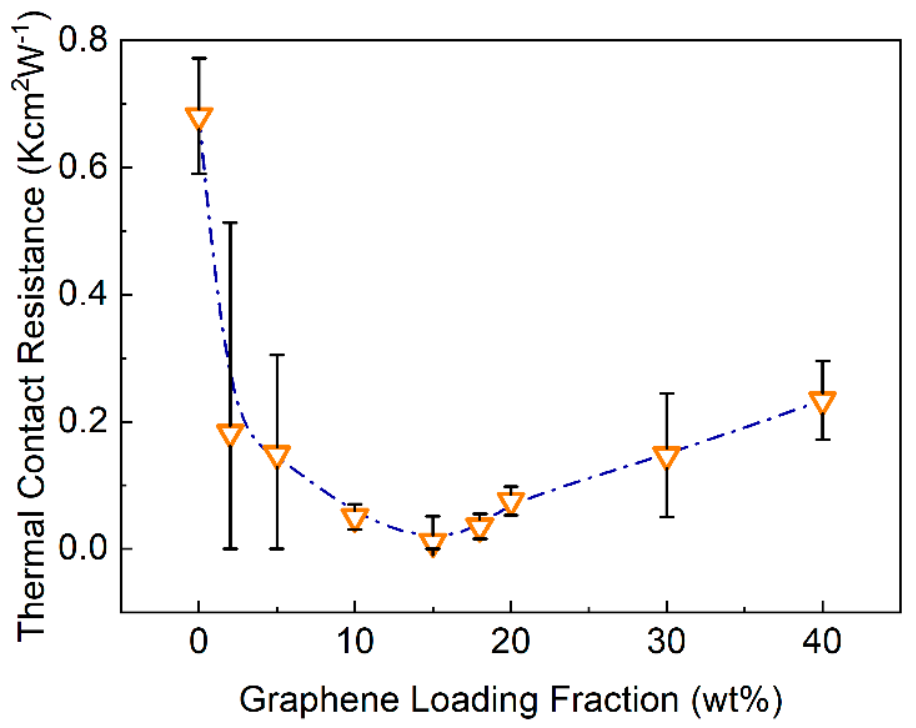

Figure 5. Thermal contact resistance, $R_{C}$, of TIMs as a function of the graphene loading, $\xi$. Note the non-monotonic dependence of $R_{C}$ on graphene loading. The bars show the standard error of the linear fittings used for data extraction.

Assuming that the "bulk" thermal conductivity of the TIM layer in semi-solid or semi-liquid TIMs is much smaller than that of the binding surfaces, the contact resistance can be described using the semi-empirical model as [57,61,62]:

$$
R_{C 1+C 2}=2 R_{C}=c\left(\frac{S_{q}}{K_{T I M}}\right)\left(\frac{G}{P}\right)^{n},
$$

where $G=\sqrt{G^{2}+G^{\prime \prime}}$. Here, $G^{\prime}$ and $G^{\prime \prime}$ are considered to be the storage modulus and the loss shear modulus of TIM, $P$ is the applied pressure, $S_{q}$ is the average roughness of the two binding surfaces, and $c$ and $n$ are empirical coefficients, respectively. The two parameters of $K_{T I M}$ and $G$ have opposite effects on the contact resistance, $R_{C}$, at a constant applied pressure. Increasing the graphene filler loading would result in an increase in both $K_{T I M}$ and $G$ of the TIM layer. The equation also suggests that for TIMs with a specific 
filler, there exists an optimum filler loading where the thermal conductivity, $K_{T I M}$, would increase significantly while slightly effecting the thermal contact resistance. Combining Equations (2) and (3), one can write the total thermal resistance as:

$$
R_{t o t}=\left(\frac{1}{K_{T I M}}\right)\left\{B L T+c S_{q}\left(\frac{G}{P}\right)^{n}\right\} .
$$

In this form, the equation indicates clearly that an increase in the TIM thermal conductivity, $K_{\text {TIM }}$, can results in a reduction of the total thermal resistance.

To investigate the effect of the surface roughness on the thermal contact resistance with graphene TIMs, we measured the total thermal resistance, $R_{\text {tot }}$, of specially prepared copper plates with varying degree of roughness, $S_{q}$, using TIM tester (see Figure 2 and the Section 4). The samples were placed between the TIM tester's heat sink and source which are made of very flat steel plates. A fraction of a droplet of silicone oil was added between the top and bottom copper plates with the heat sink and source to minimize the contact resistance between the copper and steel solid-solid interfaces. Note that in this case, the total thermal resistance, assuming a one-dimensional heat transport would be:

$$
R_{\text {tot }}=B L T / K_{T I M}+2\left(R_{C, S t-o i l}+R_{C, o i l-C u}+L_{o i l} / K_{o i l}+L_{C u} / K_{C u}+R_{C, T I M-C u}\right) .
$$

In this equation, $R_{C}$ is the thermal contact resistance between various interfaces defined by the subscripts. $L$ and $K$ are the thickness and bulk thermal conductivity of different components. The subscripts "St", "Cu", "oil", and TIM, represent the steel, i.e., the heat source and sink of the TIM tester, copper plates, silicone oil, and TIM layer, respectively. We used TIMs with $\xi=15 \mathrm{wt} \%$ and $\xi=30 \mathrm{wt} \%$ to study the effects of roughness. We selected these two filler concentrations since at $\xi=15 \mathrm{wt} \%$ the minimum in $R_{C}$ is attained whereas $\xi=30 \mathrm{wt} \%$ provides a trade-off between the contact resistance and thermal conductivity-somewhat larger $R_{C}$ (see Figure 5) but enhanced $K_{T I M}$ as well (see Figure 4).

In Figure 6, we present the results of the total thermal resistance, $R_{t o t}$, of noncuring graphene TIMs dispersed between two copper plates as a function of TIM's BLT for two different graphene loadings, $\xi$, and four different values of roughness, $S_{q}$. For all the roughness values of copper plates and filler loadings, $R_{\text {tot }}$ increases with increasing $B L T$, as expected. This means that TIMs were dispersed properly without leaving unfilled air gaps. An interesting observation is that in some cases, the proper selection of BLT and graphene loading, $\xi$, can compensate for substantial increase in the roughness, $S_{q}$. Consider the case of TIM with $\xi=30 \mathrm{wt} \%$ of graphene fillers and two roughness values $S_{q}=1.2 \mu \mathrm{m}$ (purple triangle symbols) and $S_{q}=3.1 \mu \mathrm{m}$ (violet hexagon symbols). The use of $B L T \sim 300 \mu \mathrm{m}$ with the copper plates characterized by larger values of roughness, $S_{q}=3.1 \mu \mathrm{m}$, did not result in the overall increase in $R_{\text {tot }}$ as compared to the copper plates with $S_{q}=1.2 \mu \mathrm{m}$. The thermal resistance remained at $R_{t o t} \sim 2 \mathrm{Kcm}^{2} \mathrm{~W}^{-1}$ (see Figure 6).

We extracted the $y$-intercept, $b$, of each data set presented in Figure 6 from the linear regression fittings, and related that to the total thermal contact resistance of the TIMs as a function of surface roughness. According to Equation (5), for sandwiched structures, the $y$-intercept of the plot is equal to:

$$
b=2\left(R_{C, S t-o i l}+R_{C, o i l-C u}+L_{o i l} / K_{o i l}+L_{C u} / K_{C u}+R_{C, T I M-C u}\right) .
$$

The thermal resistance of the copper plates is negligible $\left(2 L_{C u} / K_{C u} \sim 7.3 \times 10^{-4} \mathrm{Kcm}^{2} \mathrm{~W}^{-1}\right)$. Therefore, the $y$-intercept of the graph in fact presents the summation of the total contact resistance of the sandwich structure, $R_{C, t o t}=2\left(R_{C, S t-o i l}+R_{C, o i l-C u}+R_{C, T I M-C u}\right)$ plus the thermal resistance of the silicone oil layers at the copper-steel interfaces $\left(R_{\text {oil }}=2 L_{\text {oil }} / K_{\text {oil }}\right)$. In each measurement, the $R_{C, S t-o i l}$, $R_{C, o i l-C u}$, and $L_{\text {oil }} / K_{\text {oil }}$ are fixed values since the roughness of upper surfaces of all the copper plates at the interfaces with the heat source and sink and the thickness of the oil 
layer are the same. Therefore, the extracted values for the $R_{C, t o t}+R_{o i l}$ presented in Figure 7 indicate a measure for evaluating the contact resistance between the TIM layer and varying roughness of the copper plates. The determined values of $R_{C, t o t}+R_{\text {oil }}$ as the function of the surface roughness, $S_{q}$, are shown in Figure 7 and listed in Table S2. As seen, $R_{C, t o t}+R_{\text {oil }}$ grows with the surface roughness which indicates an increase in $R_{C, t o t}\left(R_{o i l}\right.$ is a fixed value). The contact resistance for TIM with the higher loading, $\xi=30 \mathrm{wt} \%$, is larger than that with $\xi=15 \mathrm{wt} \%$. This is an expected trend for the oil-based noncuring TIMs as the loading fraction of fillers increases. The obtained results can help in optimization of TIM composition for applications with different surfaces, particularly those characterized by large roughness.

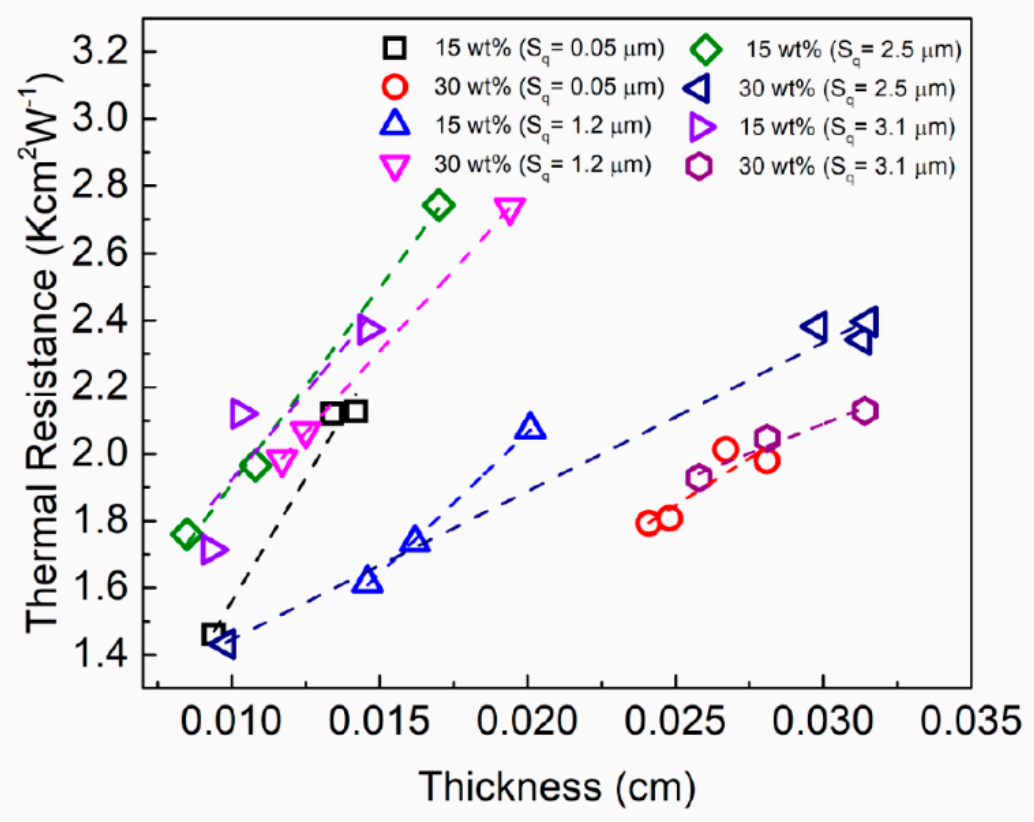

Figure 6. Thermal resistance, $R_{\text {tot }}$, of the graphene TIM dispersed between two copper plates as a function of the bond line thickness, BLT. The results are presented for two different graphene loadings, $\xi$, and four different values of roughness, $S_{q}$. In each measurement, the two copper plates used had the same roughness. The dashed lines show the linear regression fittings to the experimental data.

In high power electronic packaging, e.g., insulated gate bipolar transistors (IGBT) or silicon carbide-based device, a non-curing TIM layer is typically applied between the direct bond copper (DBC) layer and the heat sink [21,63-65]. This layer and its performance reliability [66-68] is usually the bottleneck of the packaging design since its thermal resistance is the highest among the other constituent components. Therefore, efforts have been focused on decreasing the thermal resistance of this layer by enhancing the bulk thermal conductivity of the TIM and reducing the BLT at the interface. By reducing the BLT layer, the effect of the contact resistance and roughness of the adjoining surfaces become more dominant. Recent endeavors towards application of diamond-based electronics improves the heat transport at device level owing to the high thermal conductivity of diamond. However, it still lacks proper treatment and dissipation of the generated heat at the system and packaging level where the high roughness of the diamond-based devices become problematic. Our results show that the change of roughness in the scale of $\sim 1 \mu \mathrm{m}$ substantially increases the thermal contact resistance by a factor of $\times 2$ and hence, should be addressed properly in the packaging process. Our results also suggest that graphene-based TIMs with optimized filler loading can be a potential solution for high-power electronics owing to their improved thermal conductivity and low thermal contact resistance. 


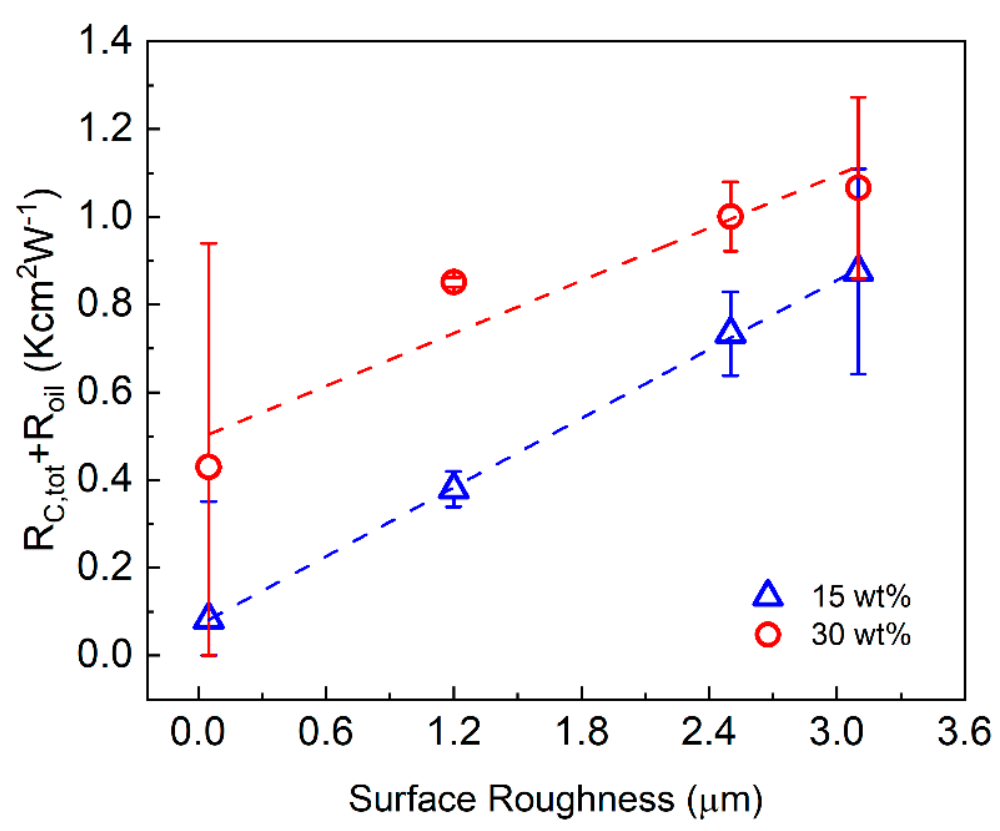

Figure 7. $R_{C, t o t}+R_{o i l}$, of graphene TIMs between two copper surfaces as a function of the surface roughness, $S_{q}$.

\section{Conclusions}

We investigated the thermal contact resistance of the noncuring graphene TIMs with the surfaces characterized by various degrees of roughness. It was found that the thermal contact resistance depends on the graphene loading non-monotonically, achieving its minimum at the loading fraction of $\sim 15 \mathrm{wt} \%$ for the studied mixture of graphene fillers. Increasing the surface roughness by $1 \mu \mathrm{m}$ results in the approximately factor of $\times 2$ increase in the thermal contact resistance, $R_{C}$, for these TIMs. The total thermal resistance of the layer of the noncuring thermal interface material scales linearly with the bond-line thickness in the studies range from 5 to $35 \mu \mathrm{m}$. A projection to the micrometer bond-line thicknesses indicate that graphene thermal interface materials can meet the thermal management requirements for the high-power electronics. The obtained results are important for thermal management of high-power electronics implemented with diamond and other wide-band-gap semiconductors, which are typically characterized by a large degree of interface roughness.

\section{Methods}

Material synthesis: Noncuring TIMs with graphene fillers were prepared from commercial FLG flakes (xGNP H-25, XG Sciences, Lansing, MI, USA, NAM) with the vendor specified average lateral dimension of $\sim 25 \mu \mathrm{m}$. The mixture of graphene and FLG was weighed in a cylindrical container to obtain the desired filler concentration in each TIM sample. To maintain the quality and size of the fillers, acetone was added, thus ensuring that the fillers are not agglomerated during the mixing process [69]. The mixture of graphene-FLG fillers with acetone was introduced to silicone oil (Fisher Scientific, Hampton, NH, USA, NAM) base polymer, also known as PDMS-Poly(dimethylsiloxane). The weighing of each component was performed using the professional scale (Ohaus Corporation, Parsippany, NJ, USA, NAM). The resulting compound was then mixed using a high-speed shear mixer (Flacktek Inc., Landrum, SC, USA) at the speed setting of $300 \mathrm{rpm}$ for $20 \mathrm{~min}$. The role of the solvent, acetone, was to assist in obtaining the homogenous dispersion of the fillers in the base polymer. At the next step, acetone was evaporated in an oven (Across International, Livingston, NJ, USA, NAM) at $\sim 70{ }^{\circ} \mathrm{C}$ for $2 \mathrm{~h}$ to ensure that it does not remain in the final TIM. The described method of TIM preparation is a modification of the procedure reported by some of us previously [41]. 
Surface roughness: For this study, we used copper square plates (Midwest Steel Supply, Rogers, MN, USA, NAM) of thickness $1.09 \mathrm{~mm}$ and dimensions of $1 \times 1 \mathrm{in}$. The copper plates were polished to different degree of roughness using the Metprep 3 polisher (Allied High-Tech Products, Inc., Compton, CA, USA, NAM). The copper plates were polished with the 8-inch, 180 grit silicon carbide paper discs (Allied High-Tech Products, Inc). A 3D optical profilometer (Profilm 3D, Filmetrics Inc., San Diego, CA, USA, NAM) was used to quantitatively determine the surface roughness of the copper plates. The optical profiler operates on the basis of a non-contact optical technique of the white-light interferometry (WLI) [53]. A 50× Nikon Mirau interferometric objective lens was used to determine the surface profile of the plates. The $S_{q}$ roughness was defined as [70]:

$$
S_{q}=\sqrt{\frac{1}{A} \int^{A} \int^{2} Z^{2}(x, y) d x d y}
$$

Here, $A$ is the area and $Z(x, y)$ is the surface profile amplitude. The $50 \times$ lens has been chosen to improve the accuracy of the data acquisition process.

Thermal Characterization: The thermal conductivity and thermal contact resistance of the TIMs were measured using the industrial TIM tester (LongWin Science and Technology Corp, Taiwan, Asia). The tester utilizes the steady-state method and meets the requirements of the industry standard ASTM D5470-06. The noncuring graphene TIMs were tested under the pressure of $80 \mathrm{psi}$ and a temperature of $80{ }^{\circ} \mathrm{C}$ for $40 \mathrm{~min}$ for each thickness. The plastic shims (Precision Brand Products Inc., Downers Grove, IL, USA, NAM) were used to measure the graphene TIMs at different bond line thicknesses (BLT). The temperature at the heat source was constant for all measurements of TIMs at all measured BLT. TIMs were placed and tested in between the heat source and heat sink and is subjected to a temperature gradient at a set uniform pressure load. The pressure and temperature were constant to measure the thermal properties of TIMs. The measurements allowed us to determine the thermal conductivity and thermal contact resistance of each TIM [54]. The thermal properties of the TIMs were measured with the steel plates provided with the TIM tester. To determine the effect of surface roughness on the thermal contact resistance of TIMs, the instrument was calibrated for proper thickness using the copper plates with the same roughness. To avoid the presence of air gaps between the plates of the TIM tester and the copper plates an ultra-thin layer of silicone oil was used on each side. This process was consistently repeated for all sets of copper plates. The TIMs were then sandwiched between the copper plates. The plastic shims ensured the desired BLT. The constant test conditions were maintained during the testing of the TIMs with and without the copper plates. Additional details are provided in the Supplementary Materials.

Supplementary Materials: The following are available online at https:/ / www.mdpi.com/article/ 10.3390/nano11071699/s1, Figure S1: Schematic of LongWin ASTM D5470-06 TIM Tester used in this study to measure the thermal resistance of TIM applied between two metal plates. This standard follows the one-dimensional heat conduction measurement technique. Heat flows from the heating plate near the heating source to the cooling plate near the cooling unit. Table S1: Thermal conductivity and thermal contact resistance of graphene TIMs. Table S2: Thermal contact resistance of graphene TIMs with different plate roughness.

Author Contributions: A.A.B. and F.K. conceived the idea of the study and coordinated the project; S.S. prepared the thermal compounds, performed sample characterization, thermal conductivity measurements, thermal contact measurements, surface roughness measurements and conducted the data analysis; F.K. contributed to the experimental and theoretical data analysis; A.A.B. contributed to the thermal data analysis; F.K. and A.A.B. led the manuscript preparation; all authors contributed to the writing and editing of the manuscript. All authors have read and agreed to the published version of the manuscript.

Funding: This work was supported, in part, by ULTRA, an Energy Frontier Research Center (EFRC) funded by the U.S. Department of Energy, Office of Science, Basic Energy Sciences un- 
der Award \# DE-SC0021230. The surface roughness measurements were performed in the UCR Nanofabrication Facility.

Institutional Review Board Statement: Not Applicable.

Informed Consent Statement: Not Applicable.

Data Availability Statement: Not Applicable.

Conflicts of Interest: The authors declare no conflict of interest.

\section{References}

1. Huang, W.; Stan, M.R.; Gurumurthi, S.; Ribando, R.J.; Skadron, K. Interaction of Scaling Trends in Processor Architecture and Cooling. In Proceedings of the 26th Annual IEEE Semiconductor Thermal Measurement and Management Symposium (SEMI-THERM), Santa Clara, CA, USA, 21-25 February 2010; pp. 198-204.

2. Ferain, I.; Colinge, C.A.; Colinge, J.-P. Multigate Transistors as the Future of Classical Metal-Oxide-Semiconductor Field-Effect Transistors. Nature 2011, 479, 310-316. [CrossRef] [PubMed]

3. Schelling, P.K.; Shi, L.; Goodson, K.E. Managing Heat for Electronics. Mater. Today 2005, 8, 30-35. [CrossRef]

4. Haensch, W.; Nowak, E.J.; Dennard, R.H.; Solomon, P.M.; Bryant, A.; Dokumaci, O.H.; Kumar, A.; Wang, X.; Johnson, J.B.; Fischetti, M.V. Silicon CMOS Devices Beyond Scaling. IBM J. Res. Dev. 2006, 50, 339-361. [CrossRef]

5. Bar-Cohen, A.; Matin, K.; Narumanchi, S. Nanothermal Interface Materials: Technology Review and Recent Results. J. Electron. Packag. 2015, 137, 040803. [CrossRef]

6. Yan, Z.; Liu, G.; Khan, J.M.; Balandin, A.A. Graphene Quilts for Thermal Management of High-Power GaN Transistors. Nat. Commun. 2012, 3, 827. [CrossRef] [PubMed]

7. Khanna, V.K. Diamond electronics for ultra-hot environments. In Extreme-Temperature and Harsh-Environment Electronics Physics, Technology and Applications; IOP Publishing: Bristol, UK, 2017; ISBN 9780750311557.

8. Kim, D.; Yamamoto, Y.; Nagao, S.; Wakasugi, N.; Chen, C.; Suganuma, K. Measurement of Heat Dissipation and ThermalStability of Power Modules on DBC Substrates with Various Ceramics by SiC Micro-Heater Chip System and Ag Sinter Joining. Micromachines 2019, 10, 745. [CrossRef]

9. Warzoha, R.; Wilson, A.; Donovan, B.; Donmezer, N.; Giri, A.; Hopkins, P.; Choi, S.; Pahinkar, D.; Shi, J.; Graham, S.; et al. Applications and Impacts of Nanoscale Thermal Transport in Electronics Packaging. J. Electron. Packag. 2021, 143, 020804. [CrossRef]

10. Faili, F.N.; Engdahl, C.; Francis, E. GaN-on-Diamond Substrates for HEMT Applications. Diam. Tools J. $2009,1,52-55$.

11. Hamann, H.F.; Weger, A.; Lacey, J.A.; Hu, Z.; Bose, P.; Cohen, E.; Wakil, J. Hotspot-Limited Microprocessors: Direct Temperature and Power Distribution Measurements. IEEE J. Solid-State Circuits 2007, 42, 56-65. [CrossRef]

12. Amalu, E.H.; Ekere, N.N.; Bhatti, R.S. High Temperature Electronics: R\&D Challenges and Trends in Materials, Packaging and Interconnection Technology. In Proceedings of the 2009 2nd International Conference on Adaptive Science \& Technology (ICAST), Accra, Ghana, 14-16 January 2009; pp. 146-153.

13. Cengel, Y.A. Heat Transfer: A Practical Approach, 2nd ed.; McGraw-Hill: New York, NY, USA, 2004.

14. Yovanovich, M.M.; Marotta, E.E. Thermal Spreading and Contact Handbook. In Heat Transfer Handbook; Bejan, A., Kraus, A.D., Eds.; Wiley: Hoboken, NJ, USA, 2003.

15. Madhusudana, C.V. Thermal Contact Conductance; Springer: New York, NY, USA, 1996.

16. Gwinn, J.P.; Webb, R.L. Performance and Testing of Thermal Interface Materials. Microelectron. J. 2003, 34, 215-222. [CrossRef]

17. Bar-Cohen, A.; Matin, K.; Jankowski, N.; Sharar, D. Two-Phase Thermal Ground Planes: Technology Development and Parametric Results. J. Electron. Packag. 2015, 137. [CrossRef]

18. Prasher, R. Thermal Interface Materials: Historical Perspective, Status, and Future Directions. Proc. IEEE 2006, 94, 1571-1586. [CrossRef]

19. Shahil, K.M.F.; Balandin, A.A. Graphene-Multilayer Graphene Nanocomposites as Highly Efficient Thermal Interface Materials. Nano Lett. 2012, 12, 861-867. [CrossRef]

20. Shahil, K.M.F.; Balandin, A.A. Thermal Properties of Graphene and Multilayer Graphene: Applications in Thermal Interface Materials. Solid State Commun. 2012, 152, 1331-1340. [CrossRef]

21. Narumanchi, S.; Mihalic, M.; Kelly, K.; Eesley, G. Thermal Interface Materials for Power Electronics Applications. In Proceedings of the 2008 11th IEEE Intersociety Conference on Thermal and Thermomechanical Phenomena in Electronic Systems, I-THERM, Orlando, FL, USA, 28-31 May 2008; pp. 395-404.

22. Liu, W.L.; Shamsa, M.; Calizo, I.; Balandin, A.A.; Ralchenko, V.; Popovich, A.; Saveliev, A. Thermal Conduction in Nanocrystalline Diamond Films: Effects of the Grain Boundary Scattering and Nitrogen Doping. Appl. Phys. Lett. 2006, 89. [CrossRef]

23. Shamsa, M.; Ghosh, S.; Calizo, I.; Ralchenko, V.; Popovich, A.; Balandin, A.A. Thermal Conductivity of Nitrogenated Ultrananocrystalline Diamond Films on Silicon. J. Appl. Phys. 2008, 103, 083538. [CrossRef]

24. Balandin, A.A.; Ghosh, S.; Bao, W.; Calizo, I.; Teweldebrhan, D.; Miao, F.; Lau, C.N. Superior Thermal Conductivity of Single-Layer Graphene. Nano Lett. 2008, 8, 902-907. [CrossRef] 
25. Balandin, A.A. Thermal Properties of Graphene and Nanostructured Carbon Materials. Nat. Mater. 2011, 10, 569581. [CrossRef] [PubMed]

26. Nika, D.L.; Balandin, A.A. Two-Dimensional Phonon Transport in Graphene. J. Phys. Condens. Matter 2012, 24, 233203. [CrossRef]

27. Ghosh, S.; Bao, W.; Nika, D.L.; Subrina, S.; Pokatilov, E.P.; Lau, C.N.; Balandin, A.A. Dimensional Crossover of Thermal Transport in Few-Layer Graphene. Nat. Mater. 2010, 9, 555-558. [CrossRef]

28. Malekpour, H.; Ramnani, P.; Srinivasan, S.; Balasubramanian, G.; Nika, D.L.; Mulchandani, A.; Lake, R.K.; Balandin, A.A. Thermal Conductivity of Graphene with Defects Induced by Electron Beam Irradiation. Nanoscale 2016, 8, 14608-14616. [CrossRef]

29. Nika, D.L.; Balandin, A.A. Phonons and Thermal Transport in Graphene and Graphene-Based Materials. Rep. Prog. Phys. 2017, 80, 036502. [CrossRef]

30. Balandin, A.A. Phononics of Graphene and Related Materials. ACS Nano 2020, 14, 5170-5178. [CrossRef]

31. Goyal, V.; Balandin, A.A. Thermal Properties of the Hybrid Graphene-Metal Nano-Micro-Composites: Applications in Thermal Interface Materials. Appl. Phys. Lett. 2012, 100, 073113. [CrossRef]

32. Yu, W.; Xie, H.; Chen, L.; Zhu, Z.; Zhao, J.; Zhang, Z. Graphene Based Silicone Thermal Greases. Phys. Lett. A 2014, 378, 207-211. [CrossRef]

33. Goli, P.; Legedza, S.; Dhar, A.; Salgado, R.; Renteria, J.; Balandin, A.A. Graphene-Enhanced Hybrid Phase Change Materials for Thermal Management of Li-Ion Batteries. J. Power Sources 2014, 248, 37-43. [CrossRef]

34. Renteria, J.; Legedza, S.; Salgado, R.; Balandin, M.P.; Ramirez, S.; Saadah, M.; Kargar, F.; Balandin, A.A. MagneticallyFunctionalized Self-Aligning Graphene Fillers for High-Efficiency Thermal Management Applications. Mater. Des. 2015, 88, 214-221. [CrossRef]

35. Saadah, M.; Hernandez, E.; Balandin, A.A. Thermal Management of Concentrated Multi-Junction Solar Cells with GrapheneEnhanced Thermal Interface Materials. Appl. Sci. 2017, 7, 589. [CrossRef]

36. Kargar, F.; Barani, Z.; Salgado, R.; Debnath, B.; Lewis, J.S.; Aytan, E.; Lake, R.K.; Balandin, A.A. Thermal Percolation Threshold and Thermal Properties of Composites with High Loading of Graphene and Boron Nitride Fillers. ACS Appl. Mater. Interfaces 2018, 10, 37555-37565. [CrossRef] [PubMed]

37. Lewis, J.S.; Barani, Z.; Magana, A.S.; Kargar, F.; Balandin, A.A. Thermal and Electrical Conductivity Control in Hybrid Composites with Graphene and Boron Nitride Fillers. Mater. Res. Express 2019, 6, 085325. [CrossRef]

38. Barani, Z.; Mohammadzadeh, A.; Geremew, A.; Huang, C.Y.; Coleman, D.; Mangolini, L.; Kargar, F.; Balandin, A.A. Thermal Properties of the Binary-Filler Hybrid Composites with Graphene and Copper Nanoparticles. Adv. Funct. Mater. 2019, 30, 1904008. [CrossRef]

39. Kargar, F.; Barani, Z.; Balinskiy, M.; Magana, A.S.; Lewis, J.S.; Balandin, A.A. Dual-Functional Graphene Composites for Electromagnetic Shielding and Thermal Management. Adv. Electron. Mater. 2019, 5, 1800558. [CrossRef]

40. Barani, Z.; Kargar, F.; Mohammadzadeh, A.; Naghibi, S.; Lo, C.; Rivera, B.; Balandin, A.A. Multifunctional Graphene Composites for Electromagnetic Shielding and Thermal Management at Elevated Temperatures. Adv. Electron. Mater. 2020, 6, 2000520. [CrossRef]

41. Naghibi, S.; Kargar, F.; Wright, D.; Huang, C.Y.T.; Mohammadzadeh, A.; Barani, Z.; Salgado, R.; Balandin, A.A. Noncuring Graphene Thermal Interface Materials for Advanced Electronics. Adv. Electron. Mater. 2020, 6, 1901303. [CrossRef]

42. Lewis, J.S.; Perrier, T.; Mohammadzadeh, A.; Kargar, F.; Balandin, A.A. Power Cycling and Reliability Testing of Epoxy-Based Graphene Thermal Interface Materials. J. Carbon Res. 2020, 6, 26. [CrossRef]

43. Fu, Y.; Hansson, J.; Liu, Y.; Chen, S.; Zehri, A.; Samani, M.K.; Wang, N.; Ni, Y.; Zhang, Y.; Zhang, Z.-B.; et al. Graphene Related Materials for Thermal Management. 2D Materials 2020, 7, 012001. [CrossRef]

44. Lewis, J.S.; Perrier, T.; Barani, Z.; Kargar, F.; Balandin, A.A. Thermal Interface Materials with Graphene Fillers: Review of the State of the Art and Outlook of Future Applications. Nanotechnology 2021, 32, 142003. [CrossRef] [PubMed]

45. Shi, Z.; Zhang, C.; Chen, X.-G.; Li, A.; Zhang, Y.-F. Thermal, Mechanical and Electrical Properties of Carbon Fiber Fabric and Graphene Reinforced Segmented Polyurethane Composites. Nanomaterials 2021, 11, 1289. [CrossRef]

46. di Pierro, A.; Mortazavi, B.; Noori, H.; Rabczuk, T.; Fina, A. A Multiscale Investigation on the Thermal Transport in Polydimethylsiloxane Nanocomposites: Graphene vs. Borophene. Nanomaterials 2021, 11, 1252. [CrossRef]

47. Liang, W.; Ge, X.; Ge, J.; Li, T.; Zhao, T.; Chen, X.; Zhang, M.; Ji, J.; Pang, X.; Liu, R. Three-Dimensional Heterostructured Reduced Graphene Oxide-Hexagonal Boron Nitride-Stacking Material for Silicone Thermal Grease with Enhanced Thermally Conductive Properties. Nanomaterials 2019, 9, 938. [CrossRef]

48. Gao, J.; Yan, Q.; Tan, X.; Lv, L.; Ying, J.; Zhang, X.; Yang, M.; Du, S.; Wei, Q.; Xue, C.; et al. Surface Modification Using Polydopamine-Coated Liquid Metal Nanocapsules for Improving Performance of Graphene Paper-Based Thermal Interface Materials. Nanomaterials 2021, 11, 1236. [CrossRef]

49. Russo, P.; Cimino, F.; Tufano, A.; Fabbrocino, F. Thermal and Quasi-Static Mechanical Characterization of Polyamide 6-Graphene Nanoplatelets Composites. Nanomaterials 2021, 11, 1454. [CrossRef]

50. Dmitriev, A.S.; Valeev, A.R. Graphene Nanocomposites as Thermal Interface Materials for Cooling Energy Devices. J. Phys. Conf. Ser. 2017, 891, 012359. [CrossRef]

51. Wang, Z.-G.; Lv, J.-C.; Zheng, Z.-L.; Du, J.-G.; Dai, K.; Lei, J.; Xu, L.; Xu, J.-Z.; Li, Z.-M. Highly Thermally Conductive GrapheneBased Thermal Interface Materials with a Bilayer Structure for Central Processing Unit Cooling. ACS Appl. Mater. Interfaces 2021, 13, 25325-25333. [CrossRef] [PubMed] 
52. Mahadevan, B.K.; Naghibi, S.; Kargar, F.; Balandin, A.A. Non-Curing Thermal Interface Materials with Graphene Fillers for Thermal Management of Concentrated Photovoltaic Solar Cells. J. Carbon Res. 2019, 6, 2. [CrossRef]

53. di Novo, N.G.; Cantu, E.; Tonello, S.; Sardini, E.; Serpelloni, M. Support-Material-Free Microfluidics on an Electrochemical Sensors Platform by Aerosol Jet Printing. Sensors 2019, 19, 1842. [CrossRef]

54. Goel, N.; Anoop, T.K.; Bhattacharya, A.; Cervantes, J.A.; Mongia, R.K.; Machiroutu, S.v.; Lin, H.-L.; Huang, Y.-C.; Fan, K.-C.; Denq, B.-L.; et al. Technical Review of Characterization Methods for Thermal Interface Materials (TIM). In Proceedings of the 2008 11th IEEE Intersociety Conference on Thermal and Thermomechanical Phenomena in Electronic Systems, Singapore, 9-12 December 2008; pp. 248-258.

55. Burzo, M.G.; Raad, P.E.; Komarov, P.L.; Wicaksono, C.; Choi, T.Y. Measurement of Thermal Conductivity of Nanofluids and Thermal Interface Materials Using the Laser-Based Transient Thermoreflectance Method. In Proceedings of the 29th IEEE Semiconductor Thermal Measurement and Management Symposium, San Jose, CA, USA, 17-21 March 2013; pp. $194-199$.

56. Raza, M.A.; Westwood, A.; Stirling, C. Comparison of Carbon Nanofiller-Based Polymer Composite Adhesives and Pastes for Thermal Interface Applications. Mater. Des. 2015, 85, 67-75. [CrossRef]

57. Prasher, R.S.; Shipley, J.; Prstic, S.; Koning, P.; Wang, J. Thermal Resistance of Particle Laden Polymeric Thermal Interface Materials. J. Heat Transf. 2003, 125, 1170-1177. [CrossRef]

58. Evans, W.; Prasher, R.; Fish, J.; Meakin, P.; Phelan, P.; Keblinski, P. Effect of Aggregation and Interfacial Thermal Resistance on Thermal Conductivity of Nanocomposites and Colloidal Nanofluids. Int. J. Heat Mass Transf. 2008, 51, 1431-1438. [CrossRef]

59. Zhang, L.; Ruesch, M.; Zhang, X.; Bai, Z.; Liu, L. Tuning Thermal Conductivity of Crystalline Polymer Nanofibers by Interchain Hydrogen Bonding. RSC Adv. 2015, 5, 87981-87986. [CrossRef]

60. Mu, L.; Ji, T.; Chen, L.; Mehra, N.; Shi, Y.; Zhu, J. Paving the Thermal Highway with Self-Organized Nanocrystals in Transparent Polymer Composites. ACS Appl. Mater. Interfaces 2016, 8, 29080-29087. [CrossRef]

61. Prasher, R.S.; Shipley, J.; Prstic, S.; Koning, P.; Wang, J.-L. Thermal Resistance of Particle Laden Polymeric Thermal Interface Materials. In Proceedings of the ASME 2003 International Mechanical Engineering Congress and Exposition. Electronic and Photonic Packaging, Electrical Systems and Photonic Design, and Nanotechnology, Washington, DC, USA, 15-21 November 2003; pp. 431-439. [CrossRef]

62. Prasher, R.S.; Matayabas, J.C. Thermal Contact Resistance of Cured Gel Polymeric Thermal Interface Material. Phenom. Electron. Syst. Proc. Intersoc. Conf. 2004, 1, 28-35. [CrossRef]

63. Maguire, L.; Behnia, M.; Morrison, G. Systematic Evaluation of Thermal Interface Materials-a Case Study in High Power Amplifier Design. Microelectron. Reliab. 2005, 45, 711-725. [CrossRef]

64. Skuriat, R.; Li, J.F.; Agyakwa, P.A.; Mattey, N.; Evans, P.; Johnson, C.M. Degradation of Thermal Interface Materials for HighTemperature Power Electronics Applications. Microelectron. Reliab. 2013, 53, 1933-1942. [CrossRef]

65. Broughton, J.; Smet, V.; Tummala, R.R.; Joshi, Y.K. Review of Thermal Packaging Technologies for Automotive Power Electronics for Traction Purposes. J. Electron. Packag. 2018, 140, 1-11. [CrossRef]

66. Li, X.; Chen, X.; Lu, G.-Q. Reliability of High-Power Light Emitting Diode Attached with Different Thermal Interface Materials. J. Electron. Packag. 2010, 132, 031011. [CrossRef]

67. Anithambigai, P.; Shanmugan, S.; Mutharasu, D.; Zahner, T.; Lacey, D. Study on Thermal Performance of High Power LED Employing Aluminum Filled Epoxy Composite as Thermal Interface Material. Microelectron. J. 2014, 45, 1726-1733. [CrossRef]

68. Möller, E.; Middelstädt, L.; Grieger, F.; Lindemann, A.; Wilde, J. Investigation on the Suitability of Electrically Conductive Adhesives for Die-Attachment of Power Devices. In Proceedings of the European Microelectronics Packaging Conference (EMPC), Friedrichshafen, Germany, 14-16 September 2015; pp. 1-5.

69. Yu, A.; Ramesh, P.; Itkis, M.E.; Bekyarova, E.; Haddon, R.C. Graphite Nanoplatelet-Epoxy Composite Thermal Interface Materials. J. Phys. Chem. C 2007, 111, 7565-7569. [CrossRef]

70. Abbott, J.P.R.; Zhu, H. 3D Optical Surface Profiler for Quantifying Leaf Surface Roughness. Surf. Topogr. Metrol. Prop. 2019, 7, 045016. [CrossRef] 